\title{
Pre-acting control for shock and impact isolation systems
}

\author{
D.V. Balandin ${ }^{\mathrm{a}}$, N.N. Bolotnik ${ }^{\mathrm{b}}$ and W.D. Pilkey ${ }^{\mathrm{c}, *}$ \\ ${ }^{a}$ Research Institute for Applied Mathematics and Cybernetics, Nizhny Novgorod State University, 10 Uljanov St., \\ Nizhny Novgorod 603005, Russia \\ ${ }^{\mathrm{b}}$ Institute for Problems in Mechanics of the Russian Academy of Sciences, 101-1 Prospect Vernadskogo, Moscow \\ 119526, Russia \\ ${ }^{\mathrm{c}}$ Department of Mechanical and Aerospace Engineering, University of Virginia, 122 Engineer's Way, P.O. Box \\ 400746, Charlottesville, VA 22904-4746, USA
}

\begin{abstract}
Pre-acting control in shock/impact isolation systems is studied. With pre-acting control, the isolation system begins to respond to an impact before this impact has been applied to the base. The limiting performance of the isolator with pre-acting control is investigated for a single-degree-of-freedom system subject to an instantaneous impact. The isolation performance index is defined as the maximum of the absolute value of the displacement of the object to be isolated relative to the base, provided that the magnitude of the control force transmitted to the object does not exceed a prescribed value. It is shown that there is a substantial advantage in the use of pre-acting isolators over isolators without pre-action. Particular attention is given to a pre-acting isolator based on a passive elastic element (a spring) separating the object to be protected from the base. An example illustrates the calculation of the design parameters of such an isolator.
\end{abstract}

Keywords: Shock isolation, pre-acting control, optimal control, limiting performance, parametric synthesis

\section{Introduction}

Impacts are one of the most frequently occurring types of mechanical disturbances to which various engineering systems and structures can be subjected when being operated. For example, impacts occur from the action of a jackhammer, in sports or military action, or in aircraft or automobile accidents.

Shock/impact isolation is the basic concept for the protection from impact and shock disturbances. A separating medium (shock/impact isolator) is placed between the base, which is subject to a shock/impact loading, and the body (object) to be protected. This medium allows the object to move relative to the base, which can lead to a reduction in the peak force transmitted to the object, as compared with the case where the object is rigidly attached to the base. Simple passive shock isolators are designed with materials possessing an elastic compliance or devices consisting of springs and dashpots. In the general case, a shock isolator can be a complex controlled device with measuring sensors, control microprocessors, and actuators.

The shock isolation concept is not new and, apparently, it has been utilized since ancient times. However, a comprehensive scientific analysis and justification of this approach was undertaken only in the last century, primarily in relation to the protection of military equipment from seismic-type shock excitations during nuclear explosions. This analysis revealed a number of interesting and, at the first sight, rather unexpected characteristics. For example, the utilization of shock isolators can lead to the undesirable effect that the peak magnitude of the force transmitted to the object separated from the base by an isolation system can increase rather than decrease, as compared with the

\footnotetext{
*Corresponding author. E-mail: wdp@virginia.edu.
} 
case of rigid attachment of the object to the base. This fact has a simple explanation. The force acting on the body to be protected is proportional to its absolute acceleration. The time during which the impact force acts on the base is finite, although small. Let the mass of the object be much less than that of the base, which is frequently the case in practice. In this case, the influence of the object on the motion of the base is insignificant. Let, in addition, the impact force be constant and, hence, the base moves with constant acceleration during the action of this force. If the period of vibration of the object is less than the impact duration, then the absolute acceleration of the base and the relative acceleration of the object will coincide in direction at some time instant, and hence, the force acting on the object will exceed the force that would have acted if the object had been rigidly attached to the base. Apparently, Anastasevich [1] was the first to note this fact using a single-degree-of-freedom system. Ishlinskii [2,3] in a more thorough investigation stated and proved a theorem that associates the isolation effectiveness that can be achieved in principle with the ratio of the isolator stroke (the maximum displacement of the object relative to the base) to the distance traveled by the base for the time interval during which the acceleration of the base does not change in direction. The greater this ratio, the more effective the isolation. On the basis of this theorem, Ishlinskii [3] has formulated an engineering guideline which states that the utilization of shock isolators can be ineffective if the deceleration (or acceleration) path of the base exceeds the isolation stroke.

In the late 1950s, a new approach to the investigation of the problem of shock and vibration isolation appeared. Isolators were interpreted as control devices that generate a force between the base and the object to be protected. This approach began to unfold in 1957 when Sevin [4] explored the problem of the minimum peak response of a linear oscillator with an incompletely prescribed excitation. This is a minmax problem. After a 1959-1960 study [5] of a shock isolated floor system equipped with suspensions of various types, Sevin formulated related problems culminating in the optimum shock isolator problem in which the particular isolator configuration is replaced by a generic, time-dependent control force and the problem becomes one of open-loop control. In this problem, for example, the open-loop control (the time history of the force acting between the object and the base) is sought to minimize the peak absolute value of the displacement of the object relative to the base, provided that the absolute value of the control force does not exceed a prescribed value. Thus, this approach enables one to establish limits on possible improvements of a dynamic system in terms of prescribed criteria. These limits depend on constraints imposed on the control force and other quantities characterizing the behavior of the system. This dependence can be represented by trade-off-curves plotting the limiting values of the performance criteria, which characterize the limiting performance of the system, against the maximum values allowed for the constrained quantities. These curves can then be used by the designer of the system to evaluate the relative merits of an existing prototype and assess prospects for an improvement.

Kolovskii [6,7] initiated applications of methods of control theory to the analysis of vibration isolation systems. At about this time, Guretskii, Sevin and Pilkey [8,9] had formalized the optimal shock isolation problem based on optimal control theory and mathematical programming.

As mentioned above, the statement of this problem does not involve the design configuration of the shock isolator. Therefore, by solving this optimal control problem, one can, in principle, calculate the absolute minimum of the performance index depending only on the external disturbance, the inertial parameters of the object, and the prescribed value of the constraint. This problem was the starting point of the general concept of the limiting performance analysis of shock isolation systems.

Shock isolators normally start responding to an impact only after this impact has occurred. In fact, the impact itself serves as a signal for the isolation system to be engaged. In a number of cases, one can predict the initiation time of the impact and its characteristics ahead of time. In this case, the isolation system can start operating in advance, before the impact has occurred. Such isolation systems can be referred to as pre-acting isolation systems or isolation systems with pre-acting control. In the present paper, the limiting performance analysis of pre-acting isolators is performed for a single-degree-of-freedom system subjected to an instantaneous impact (shock) impulse. The effectiveness of such an isolator is evaluated in comparison with that of the traditional isolators. Implementation of a pre-acting shock isolator with a passive elastic isolating element (a spring) is discussed. This approach is related to the pre-acting control concept known in the control theory. To form the control signal, information about the current and previous states of the system must be available, as well as a prediction of the full behavior of the disturbance. If this prediction is reliable enough, pre-acting control can lead to considerable improvement of the system performance. 


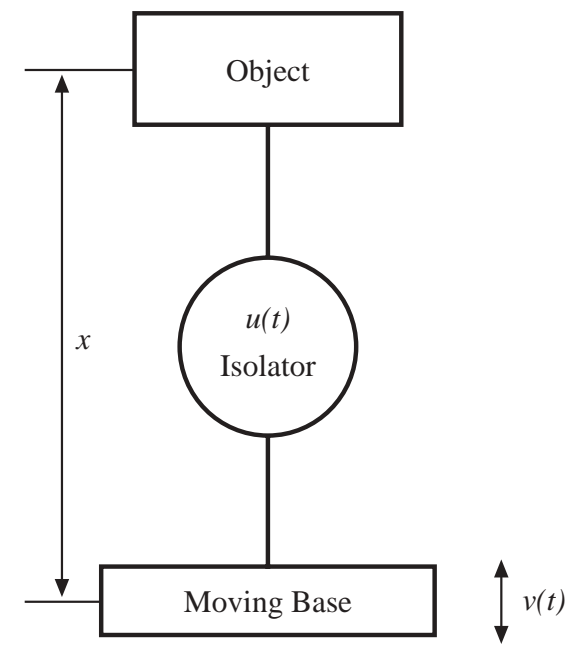

Fig. 1. The object to be isolated on a base subject to the disturbance.

For the fundamentals of shock and impact isolation, as well as a description of the design of basic types of shock/impact isolators, see, for example, the handbooks of $[10,11]$. The historical perspectives and comprehensive bibliographies of the theory of optimal shock and impact isolation are available in [12] and the monograph [13].

\section{Statement of the problem and some familiar results}

Consider a simple single-degree-of-freedom shock isolation system. The object to be protected (isolated) is connected to the base by means of a shock/impact isolator treated as a controller that generates a control force between the base and the object (Fig. 1). The base and the object are regarded as rigid bodies able to move along the same straight line. In this case, the motion of the body to be isolated relative to the base is governed by the equation

$$
m \ddot{x}+u=m v, \quad v=-\ddot{z},
$$

where $m$ is the mass of the object, $x$ is the displacement of the object relative to the base, $z$ is the displacement of the base relative to a fixed (inertial) reference frame, and $u$ is the control force applied to the base (accordingly, $-u$ is the control force applied to the object).

We will assume the acceleration of the base, $\ddot{z}$, and, hence, the variable $v$ in Eq. (1) to be prescribed functions of time characterizing the disturbance of the base. In particular, this disturbance can be induced by a shock or impact loading of the base.

Consider the following optimal control problem for the system of Eq. (1) [8,9]:

Problem 1. For the system of Eq. (1) with a prescribed function $v(t)$ and zero initial conditions

$$
x(0)=0, \quad \dot{x}(0)=0,
$$

find an optimal control $u_{0}(t)$ in the class of piecewise continuous functions that (i) satisfies the constraint

$$
\left|u_{0}(t)\right| \leqslant U
$$

where $U$ is a prescribed positive number, and (ii) minimizes the peak absolute value of the object's displacement,

$$
J\left(u_{0}\right)=\min _{u} J(u), \quad J(u)=\max _{t \in[0, \infty)}|x(t ; u)|,
$$

where $x(t ; u)$ denotes the solution of the initial value problem of Eqs (1) and (2) for the specified control $u=u(t)$.

The criterion $J(u)$ in Eq. (4) determines the necessary space between the object and the base for the control $u$. (In the engineering literature on shock isolation, this space is sometimes referred to as the rattlespace.) The inequality of Eq. (3) constrains the peak absolute value of the force exerted on the body by the isolator. The value of $U$ should 
be chosen so that the control force would not violate the operational integrity of the object. Therefore, Problem 1 could be interpreted as the problem of the minimization of the necessary space between the object and the base, provided that its operational integrity is preserved.

Problem 1 has been called the problem of the limiting capabilities of shock isolation (or the limiting performance problem), since its solution provides the absolute minimum of the performance index $J$, irrespective of the hardware implementation of the isolator. The solution of Problem 1, with the subsequent interpretation of the results in engineering terms related to the design of shock/impact isolation systems, forms the content of the limiting performance analysis of shock isolation. The limiting performance analysis may involve, in particular, a comparison of the theoretical absolute optimum of the performance index characterizing the isolation quality with the corresponding values for the existing prototypes. This comparison will allow an engineer to assess prospects for improving the operating characteristics of isolation systems by changing their design. Specific features of the control law resulting from the solution of the limiting performance problem can serve as guidelines for the further development.

The core of the limiting performance analysis is that the specific design of the isolator is not taken into account and its action is modelled by a generic control force. This control force is to be determined so as to optimize (minimize or maximize) a criterion characterizing the behavior of the system. Hence, the limiting performance problem is an optimal control problem. In many cases, for the limiting performance analysis, it is sufficient to find an open-loop optimal control, depending on time alone, rather than a feedback control, depending on all state variables of the system. This fact substantially simplifies the problem.

The concept of the limiting performance analysis for shock isolation systems was pioneered by Sevin and Pilkey [9] in USA and Guretskii and Kolovskii [7,8] in the USSR in the late 1950s - early 1960s. Subsequently, it has been developed and applied to solve important specific problems of shock isolation by a number of authors. For the modern presentation of this concept, see, for example [9,13,14].

The concept of the limiting performance analysis can be applied not only to shock/impact isolation systems but to virtually all controlled engineering systems with any number of degrees of freedom.

We will present a well-known solution of Problem 1 for the case of an instantaneous impact, as a result of which the velocity of the base, $\dot{z}$, jumps by the value $-v_{0}$. Accordingly, the relative velocity of the object to be protected instantaneously becomes equal to $v_{0}$. We assume that the impact occurs at the time instant $t=0$ coinciding with the instant at which the control starts acting. In this case, we have $v(t)=v_{0} \delta(t)$, where $\delta(t)$ is Dirac's delta function, and the problem of Eqs (1) and (2) is equivalent to the following initial value problem:

$$
m \ddot{x}+u=0, \quad x(0)=0, \quad \dot{x}(0)=v_{0} .
$$

Without loss of generality, we assume $v_{0}>0$.

The optimal control in this case is identically equal to $U$ on the time interval $0 \leqslant t \leqslant m v_{0} / U$, during which the body to be protected decelerates from the initial velocity to a complete stop at the position $x_{*}=m v_{0}^{2} /(2 U)$. For $t>m v_{0} / U$, the control is not uniquely defined: any control that satisfies the constraints $|u(t)| \leqslant U$ and $|x(t ; u)| \leqslant m v_{0}^{2} /(2 U)$ is appropriate. In particular, one can set $u(t) \equiv 0$ for $t>m v_{0} / U$. From the foregoing it follows that

$$
J\left(u_{0}\right)=\frac{m v_{0}^{2}}{2 U} .
$$

This solution is easy to obtain from an energy relationship for the system of Eq. (5). The initial conditions of Eq. (5) imply that at the initial time instant, the body has a positive relative velocity (we have assumed $v_{0}>0$ ). Since the functions $x(t)$ and $\dot{x}(t)$ are continuous, the position coordinate of the body, $x(t)$, will monotonically increase until the velocity, $\dot{x}(t)$, vanishes. Let $x_{*}$ be the point at which the velocity vanishes for the first time after the beginning of the motion. The work - kinetic energy theorem applied to the point mass, the motion of which is governed by the equation with initial conditions of Eq. (5), leads to the relation

$$
\frac{m v_{0}^{2}}{2}=\int_{0}^{x_{*}} u d x .
$$

From Eq. (7) and the inequality $u \leqslant U$, we have 


$$
\frac{m v_{0}^{2}}{2} \leqslant U x_{*}, \quad x_{*} \geqslant \frac{m v_{0}^{2}}{2 U}
$$

with equality being attained for $u \equiv U$. This leads to the relation of Eq. (6).

Along with the limiting performance problem of shock isolation, consider the problem of the construction of a feedback control $u$ to be formed in accordance with measured current values of the coordinate, $x$, and the velocity, $\dot{x}$, of the body to be protected relative to the base. A possible technique for constructing the feedback control involves the parametric optimization of a given family of functions $u(x, \dot{x}, \alpha)$, where $\alpha$ is the set of parameters to be determined. These parameters can result from the solution of an optimization problem the statement of which coincides with that of Problem 1, with the difference that the optimal control is to be found among the functions of a given family, rather than in the class of piecewise continuous functions of time. In this case, the optimal control problem is replaced by a constrained minimization of a function of a finite number of variables. This approach is sometimes referred to as the parametric synthesis of a shock isolator. Unlike the limiting performance analysis, the parametric synthesis utilizes information concerning the design of the isolator. This information is indirectly reflected in the specified structure of the dependence of the control force on the state variables, $x$ and $\dot{x}$. In general, the value of the performance index of shock isolation provided by the isolator designed by means of parametric synthesis exceeds the value characterizing the limiting performance. A measure of the success of the parametric synthesis is provided by ascertaining how close its performance is to the limiting performance.

We will give a number of examples of optimal feedback isolators designed for the instantaneous impact, $v(t)=$ $v_{0} \delta(t)$, by means of the parametric synthesis.

1. Undamped elastic isolator. The control force for this isolator has the form

$$
u=k|x|^{n} \operatorname{sign} x, \quad n \geqslant 0,
$$

where $k$ is the stiffness coefficient to be determined and $n$ is the exponent of power of the characteristic of the elastic element (a spring).

In the case under consideration, the relations of Eq. (5), governing the motion of the body to be protected relative to the base, have the form

$$
m \ddot{x}+k|x|^{n} \operatorname{sign} x=0, \quad x(0)=0, \quad \dot{x}(0)=v_{0} .
$$

In this system, the total mechanical energy is conserved and, accordingly, we have

$$
\frac{m \dot{x}^{2}}{2}+\frac{k|x|^{n+1}}{n+1}=\frac{m v_{0}^{2}}{2} .
$$

From the last relation, we find

$$
\max _{t}|x(t)|=\left[\frac{(n+1) m v_{0}^{2}}{2 k}\right]^{\frac{1}{n+1}}, \quad \max _{t}|u(t)|=\left[\frac{k^{\frac{1}{n}}(n+1) m v_{0}^{2}}{2}\right]^{\frac{n}{n+1}} .
$$

As follows from Eq. (12), the peak absolute value of the displacement of the body monotonically decreases, while the peak absolute value of the control force monotonically increases as $k$ increases. Therefore, for the optimal value of the stiffness coefficient, $k_{0}$, that minimizes the criterion $J$ of Eq. (4), the peak absolute value of the control force is equal to $U$. Accordingly, we obtain

$$
k_{0}=U\left[\frac{2 U}{(n+1) m v_{0}^{2}}\right]^{n}, \quad J\left(k_{0}\right)=\frac{m v_{0}^{2}}{2 U}(n+1) .
$$

By comparing the expressions of Eqs (6) and (13), we find

$$
\frac{J\left(k_{0}\right)-J\left(u_{0}\right)}{J\left(u_{0}\right)}=n \text {. }
$$

Hence, the relative difference between the minimum of the peak absolute value of the displacement provided by the elastic isolator with the characteristic of Eq. (9) and the absolute minimum increases as $n$ increases. In the limit as $n \rightarrow 0$, the isolator with the characteristic of Eq. (9) provides the limiting performance.

2. Damped power-law isolator without an elastic element. The control force of this isolator is expressed as 


$$
u=c|\dot{x}|^{r} \operatorname{sign} \dot{x}, \quad r \geqslant 0
$$

where $c$ is the damping coefficient to be determined and $r$ is the exponent of power of the damper characteristic.

For this isolator, the relations of Eq. (5), governing the motion of the body to be protected relative to the base, become

$$
m \ddot{x}+c|\dot{x}|^{r} \operatorname{sign} \dot{x}=0, \quad x(0)=0, \quad \dot{x}(0)=v_{0} .
$$

During the motion governed by Eq. (16), the relative velocity of the object to be protected, $\dot{x}$, does not change in sign. This follows from the continuity of the function $\dot{x}(t)$ and the fact that $\dot{x}(t) \equiv 0$ is a solution of Eq. (16). Therefore, once vanished, the velocity $\dot{x}(t)$ will remain zero. Since $v_{0}>0$, the velocity will remain positive until the object comes to a full stop. Accordingly, the displacement $x$ will monotonically increase during the entire motion.

Proceed from Eq. (16) to the equation for the phase trajectory defined by the function $\dot{x}(x)$. Apply the chain rule to differentiate this function to obtain $\ddot{x}=\dot{x}(d \dot{x} / d x)$. By substituting the last relation to Eq. (16) we arrive at the desired equation

$$
m \dot{x} \frac{d \dot{x}}{d x}+c \dot{x}^{r}=0, \quad \dot{x}(0)=v_{0} .
$$

The solution of the initial value problem of Eq. (17) leads to the relations

$$
x=\frac{m\left(v_{0}^{2-r}-\dot{x}^{2-r}\right)}{c(2-r)}, \text { if } r \neq 2 ; \quad x=\frac{m}{c} \ln \frac{v_{0}}{\dot{x}}, \text { if } r=2 .
$$

The maximum value of $x$ is attained at the instant when the velocity, $\dot{x}$, vanishes. From Eq. (18) it follows that if $r \geqslant 2$, then $x \rightarrow \infty$ as $\dot{x} \rightarrow 0$. Hence, if the damping exponent is greater than or equal to 2 , the body goes away to infinity. For $r<2$, the value of the criterion $J$ of Eq. (4) is finite and is given by

$$
J(c)=\frac{m v_{0}^{2-r}}{c(2-r)} .
$$

Since the velocity of the object monotonically decreases in the case under consideration, the maximum value of the damping force is attained at the initial time instant and is equal to $c v_{0}^{r}$. The minimum of the function $J(c)$ under the constraint $c v_{0}^{r} \leqslant U$ is attained at $c=c_{0}=U / v_{0}^{r}$ and is given by

$$
J\left(c_{0}\right)=\frac{m v_{0}^{2}}{U(2-r)} .
$$

A comparison of the expressions of Eqs (6) and (20) implies

$$
\frac{J\left(c_{0}\right)-J\left(u_{0}\right)}{J\left(u_{0}\right)}=\frac{r}{2-r}, \quad r<2 .
$$

The analysis of the expression of Eq. (21) shows that the relative difference of the quantity $J\left(c_{0}\right)$ from $J\left(u_{0}\right)$ increases as $r$ increases and that in the limit as $r \rightarrow 0$, the isolator with the characteristic of Eq. (15) provides the limiting performance of shock isolation. Note that the dependence of the damping force on the velocity in accordance with Eq. (15) for $r=0$ corresponds to dry friction.

3. Spring-and-dashpot isolator with a power-law characteristic. The control force of this isolator is represented by the sum of the expressions of Eqs (9) and (15), i.e.,

$$
u=c|\dot{x}|^{r} \operatorname{sign} \dot{x}+k|x|^{n} \operatorname{sign} x, \quad r>0, n>0
$$

The expressions of Eqs (9) and (15) are particular cases of Eq. (22) for $c=0$ and $k=0$, respectively. Shock isolators with the characteristic of Eq. (22) have been investigated in [13-15]. It has been shown that along with the isolators of Eq. (9) with $n=0$ and $k=U$ and Eq. (15) with $r=0$ and $c=U$, the limiting performance defined by Eq. (6) is provided by the isolator of Eq. (22) with the parameters

$$
r=2, n=1, c=\frac{U}{v_{0}^{2}}, k=\frac{2 U^{2}}{m v_{0}^{2}}
$$




$$
r=0, n=0, c+k=U
$$

The case of Eq. (23) corresponds to a rather widespread isolator with linear stiffness and quadratic damping, whereas that of Eq. (24) corresponds to a dry-friction damper connected in parallel with a bang-bang spring the force of elastic resistance of which is constant in magnitude and is directed against the displacement of the body to be protected.

Note also that isolators of the class of Eq. (22) provide shock isolation rather close to the limiting performance, although not coinciding with the latter. For example, the linear isolator $(r=1, n=1)$ with the coefficients $c=0.931 \mathrm{U} / v_{0}$ and $k=1.330 \mathrm{U}^{2} /\left(m v_{0}^{2}\right)$ provides $J=0.521 m v_{0}^{2} / U$, which exceeds the absolute minimum of Eq. (6) only by $4 \%[14,15]$.

\section{Limiting performance of shock isolation with allowance for the pre-acting control}

The statement of Problem 1 implies that the time instant at which the control begins is fixed and coincides with the initial instant of the disturbance. However, in a number of cases, it is possible to predict the action of the disturbance and actuate the controller in advance, which could substantially improve the isolation quality. The control engaged before the disturbance can be referred to as pre-acting control. The pre-acting control can be utilized in seismic protection systems and injury prevention systems for road and air vehicles. Consider, for example, a helicopter performing an emergency landing. Suppose that the helicopter hits the ground with a vertical velocity that is high enough to cause serious injuries to the pilot and passengers but not so high as to completely destroy the vehicle. To reduce the severity of injuries of the pilot and passengers, it is reasonable to equip helicopter seats with actively controlled impact isolators. For the case under consideration, it is possible to predict the velocity and time of landing based on the measurement of the height and the rate of descent. The situation is similar for automobiles, when, for instance, an obstacle that cannot be avoided is in the path of travel. In this case, the only means to reduce the severity of injuries to the driver and passengers is effective isolation from the impact. The time and velocity of impact can be calculated beforehand using the distance to the obstacle and the velocity of the automobile relative to the obstacle. The automobile must be equipped with appropriate sensors able to measure the distance to an object in front of the automobile and the velocity of the automobile relative to this object, as well as with a microcomputer to process this information and to form control signals for the shock isolation system.

We will formulate the problem of the limiting possibilities of shock isolation systems with the possibility of pre-acting control as follows:

Problem 2. Let the motion of the object to be protected relative to the base be governed on the time interval $\left(-t_{0}, \infty\right), t_{0} \geqslant 0$, by the differential equation

$$
m \ddot{x}+u=m v,
$$

where $v=v(t)$ is a prescribed function of time, which is identically zero for $-t_{0}<t<0$. Let this system be subject to zero initial conditions

$$
x\left(-t_{0}\right)=0, \quad \dot{x}\left(-t_{0}\right)=0 .
$$

It is necessary to find an optimal value $t_{0}^{*}$ and a piecewise continuous control $u_{0}(t)$ that is defined for $t \geqslant-t_{0}^{*}$, satisfies the constraint

$$
\left|u_{0}(t)\right| \leqslant U
$$

where $U$ is a prescribed positive constant, and minimizes the peak absolute value of the relative displacement of the object to be protected, i.e.,

$$
J\left(u_{0}, t_{0}^{*}\right)=\min _{u, t_{0}} J\left(u, t_{0}\right), J\left(u, t_{0}\right)=\max _{t \in\left[-t_{0}, \infty\right)}\left|x\left(t ; t_{0}, u\right)\right| .
$$

The notation $x\left(t ; t_{0}, u\right)$ in Eq. (28) stands for the solution of the initial value problem of Eqs (25) and (26) for a prescribed control $u=u(t)$. 
This problem is a generalization of Problem 1 of Section 2. In Problem 2, zero time $(t=0)$ corresponds to the start of the external disturbance (the time of impact) and $t_{0}$ to the time interval from the instant at which information about the forthcoming impact is received to the anticipated time of impact.

In the present paper, we will solve Problem 2 for the case of an instantaneous impact modelled by the impulse function $v(t)=v_{0} \delta(t)$. For convenience, we introduce the dimensionless variables

$$
x^{\prime}=\frac{U}{m v_{0}^{2}} x, t^{\prime}=\frac{U}{m v_{0}} t, t_{0}^{\prime}=\frac{U}{m v_{0}} t_{0}, u^{\prime}=\frac{u}{U}, J^{\prime}=\frac{U}{m v_{0}^{2}} J
$$

Substitution of these relations into Eqs (25)-(28), with $v(t)=v_{0} \delta(t)$, leads to Eqs (25)-(28) again, in which the parameters $m, v_{0}$, and $U$ are equal to unity. In what follows, we will omit the primes labeling the dimensionless variables.

To solve Problem 2, one can apply, for example, the graphical-analytical method of $[9,16]$. The solution procedure of this method is reasonably simple but somewhat cumbersome for presentation. For that reason, we choose not to describe the algorithm for the construction of the optimal control, but will simply provide the final expressions for the optimal control and the corresponding time history of the relative motion of the object to be isolated and discuss the limiting performance of the pre-acting isolator in comparison with the traditional one.

An optimal control has the form

$$
u_{0}(t)= \begin{cases}-1, & \text { if }-1 \leqslant t<-3 / 4 \\ 1, & \text { if }-3 / 4 \leqslant t<1 / 2 \\ 0, & \text { if } t \geqslant 1 / 2\end{cases}
$$

This control corresponds to a unit pre-acting time $\left(t_{0}^{*}=1\right)$.

In the case of the instantaneous impact, $v(t)=v_{0} \delta(t)$, Eq. (25) with the initial conditions of Eq. (26) is equivalent to the equation

$$
m \ddot{x}+u=0,
$$

subject to the conditions

$$
x\left(-t_{0}\right)=0, \dot{x}\left(-t_{0}\right)=0 ; \dot{x}(+0)=\dot{x}(-0)+v_{0} .
$$

The first two conditions of Eq. (32) coincide with the initial conditions of Eq. (26), while the third condition is that of impact; $\dot{x}(-0)$ and $\dot{x}(+0)$ denote the pre-impact velocity and the post-impact velocity, respectively. This condition implies that the relative velocity of the object jumps by $v_{0}$ at the time of impact.

Substitute the control of Eq. (30) into Eq. (31), where $m=1$ and $v_{0}=1$, and integrate this equation with reference to the conditions of Eq. (32) to obtain

$$
\begin{aligned}
& \dot{x}(t)= \begin{cases}t+1, & \text { if }-1 \leqslant t<-3 / 4 \\
-t-1 / 2, & \text { if }-3 / 4 \leqslant t<0 \\
1 / 2-t, & \text { if } 0 \leqslant t<1 / 2 \\
0, & \text { if } t \geqslant 1 / 2\end{cases} \\
& x(t)= \begin{cases}(t+1)^{2} / 2, & \text { if }-1 \leqslant t<-3 / 4 \\
1 / 16-(2 t+1)^{2} / 8, & \text { if }-3 / 4 \leqslant t<0 \\
1 / 16-(2 t+1)^{2} / 8+t, & \text { if } 0 \leqslant t<1 / 2 \\
1 / 16, & \text { if } t \geqslant 1 / 2\end{cases}
\end{aligned}
$$

The graphs of the functions $u_{0}(t)$ of Eq. (30), $\dot{x}(t)$ of Eq. (33), and $x(t)$ of Eq. (34) are plotted in Figs 2, 3 and 4 , respectively. From Eqs (33) and (34) it can be observed that the object moves in the direction of the impact on the time interval $-1 \leqslant t<-1 / 2$; at the instant $t=-1 / 2$, the velocity vanishes and the displacement attains a maximum $x(-1 / 2)=1 / 16$. Then the object moves in the direction opposite to that of the impact impulse on the interval $-1 / 2<t \leqslant 0$ and at the instant $t=0$, the velocity and the displacement become $\dot{x}(0)=-1 / 2$ and $x(0)=-1 / 16$, respectively. At the instant $t=0$, the object to be protected undergoes an impact and its velocity jumps from $-1 / 2$ to $1 / 2$, after which the object moves in the direction of the impact until it comes to a full stop at 


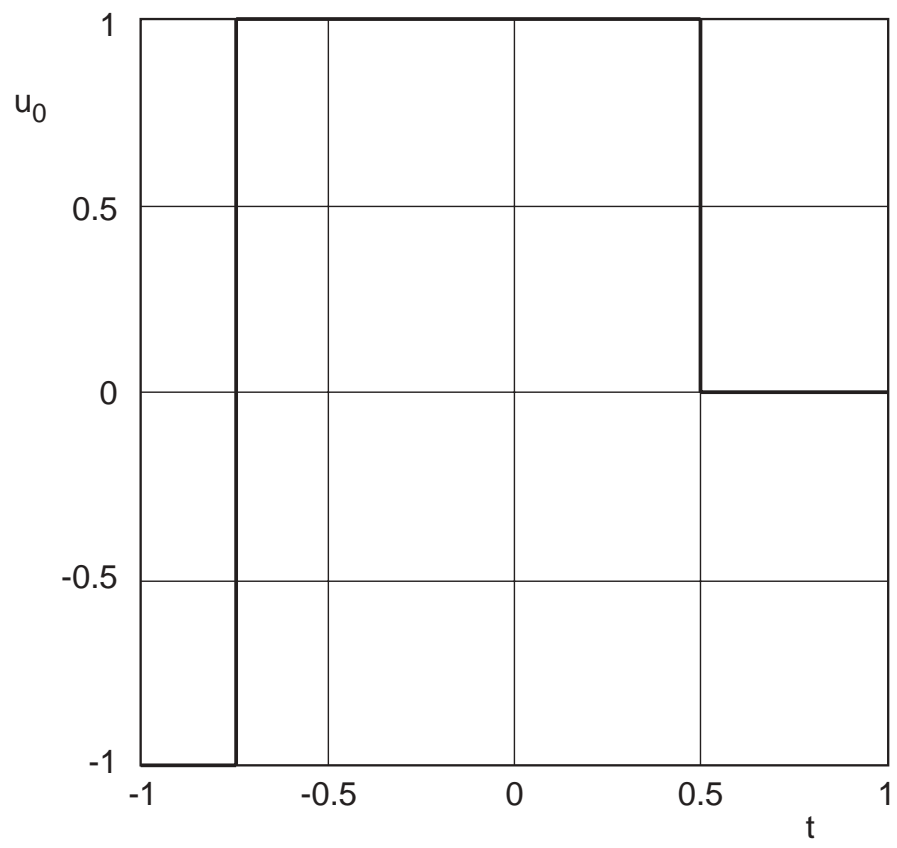

Fig. 2. Time history of the optimal pre-acting control force.

the instant $t=1 / 2$ at the position $x(1 / 2)=1 / 16$. The function $x(t)$ corresponding to the optimal motion has three points of extremum - two points of maximum (at $t=-1 / 2$ and $t=1 / 2$ ) and one point of minimum (at $t=0)$. The magnitude of the displacement at all these points of extremum is equal to $1 / 16$. Therefore, for the optimal value of the performance index of Eq. (28), in the dimensionless variables, we have

$$
J\left(u_{0}, t_{0}^{*}\right)=1 / 16, t_{0}^{*}=1 .
$$

In the original dimensional variables, the expressions of Eq. (35) have the form

$$
J\left(u_{0}, t_{0}^{*}\right)=\frac{m v_{0}^{2}}{16 U}, t_{0}^{*}=\frac{m v_{0}}{U} .
$$

A comparison of the relations of Eqs (6) and (36) shows that a pre-acting control allows a reduction in the peak magnitude of the displacement of the object to be protected by a factor of 8 relative to the absolute minimum that can be achieved without pre-action.

\section{Parametric synthesis of passive pre-acting shock isolators}

A pre-acting shock isolator can be constructed with conventional passive elements such as springs and dampers. The pre-acting operation of such isolators can be provided by pre-straining (cocking) the spring and releasing it at an appropriate time instant before the impact.

The preliminary strain of the spring and the time of its release are to be determined so as to provide the maximum effectiveness of the isolator. Consider an undamped isolator with the characteristic of Eq. (9). In this case, the motion of the object to be protected relative to the base is governed by the equation

$$
m \ddot{x}+k|x|^{n} \operatorname{sign} x=0, n \geqslant 0 .
$$

At the instant of the release of the spring, $t=-t_{0}$ (for $t_{0}$ time units before the impact), the object has been moved to the position $x=a$ (the spring has been cocked) and is in a state of rest. Accordingly, we have the initial condition

$$
x\left(-t_{0}\right)=a, \dot{x}\left(-t_{0}\right)=0 .
$$




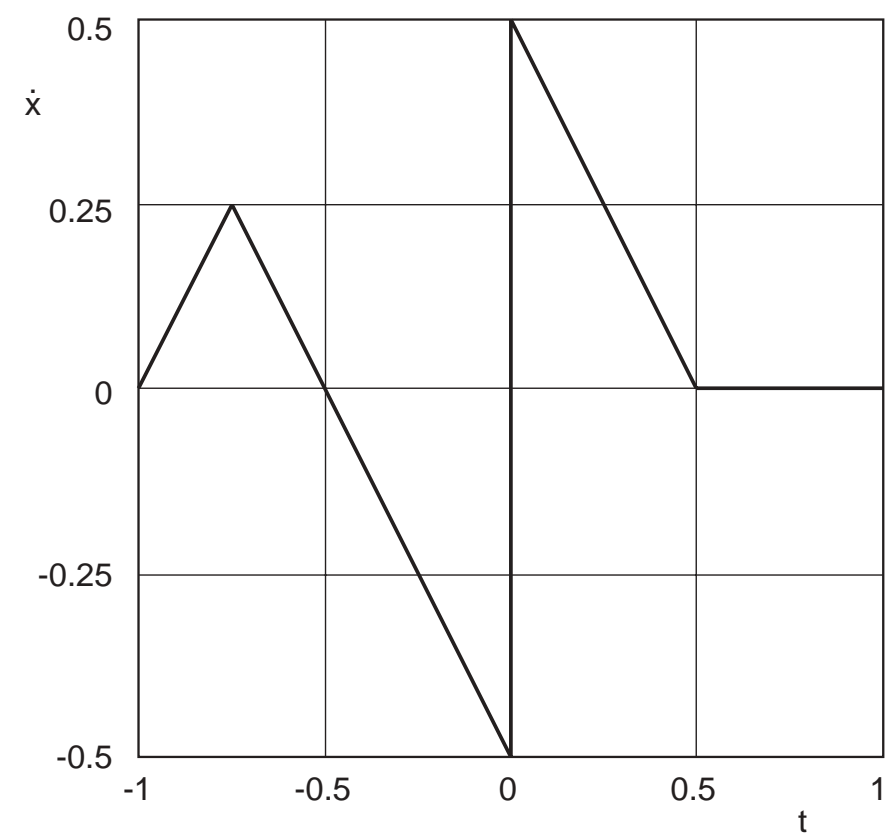

Fig. 3. Time history of the relative velocity of the object corresponding to the optimal pre-acting control.

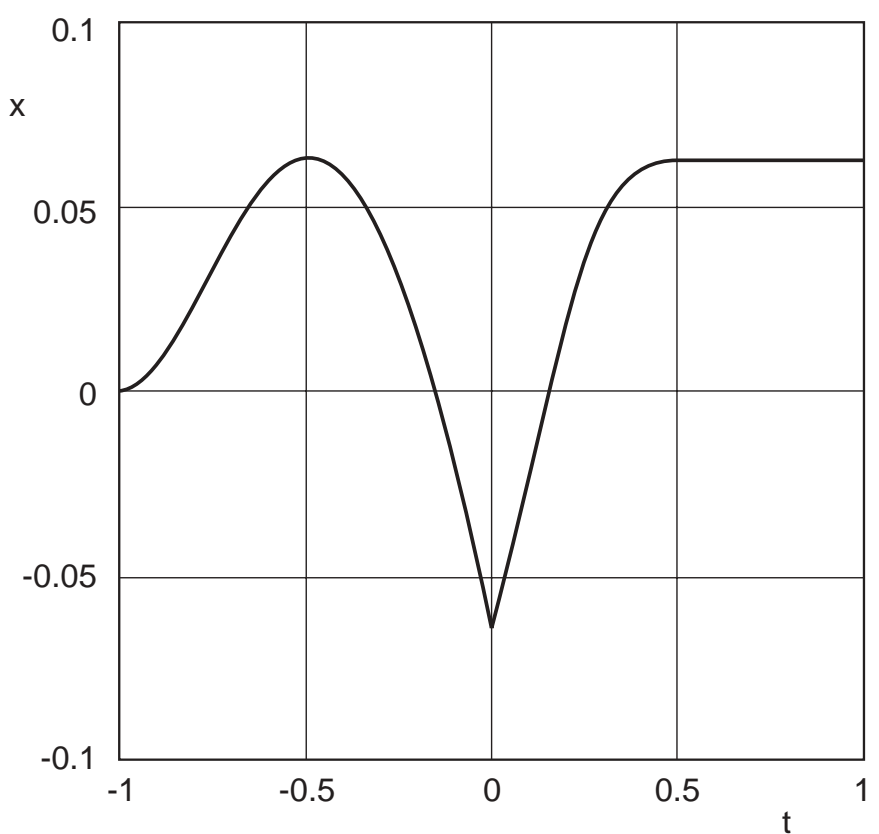

Fig. 4. Time history of the relative displacement of the object corresponding to the optimal pre-acting control.

At the impact instant $(t=0)$, the relative velocity of the object jumps by $v_{0}$, which is expressed by the additional condition

$$
\dot{x}(+0)=\dot{x}(-0)+v_{0} .
$$

As was the case in the previous sections, we define the isolation performance criterion as the peak absolute value of the displacement of the object to be protected relative to the base, 


$$
J=J\left(t_{0}, a, k\right)=\max _{\left[t_{0}, \infty\right)}|x(t)|,
$$

provided that the force transmitted to the object by the isolator (control force) does not exceed the prescribed value $U$

$$
k|x(t)|^{n} \leqslant U
$$

We will determine the optimal values of the stiffness coefficient of the spring $\left(k=k_{0}\right)$, the initial coordinate of the object $\left(a=a_{0}\right)$, and the pre-action time $\left(t_{0}=t_{0}^{*}\right)$ corresponding to the minimum of the criterion of Eq. (40) subject to the constraint of Eq. (41).

The total mechanical energy of the system of Eq. (37) is conserved on the time intervals $\left[-t_{0}, 0\right)$ and $(0, \infty)$. On the interval $\left(-t_{0}, 0\right)$,

$$
\frac{m \dot{x}^{2}}{2}+\frac{k|x|^{n+1}}{n+1}=\frac{k|a|^{n+1}}{n+1},-t_{0} \leqslant t<0 .
$$

At the time of impact, the kinetic energy of the object jumps by the value $\Delta K=m\left(\dot{x}(-0)+v_{0}\right)^{2} / 2-$ $m \dot{x}^{2}(-0) / 2=m\left(v_{0}^{2} / 2+\dot{x}(-0) v_{0}\right)$, whereas the potential energy of the spring remains unchanged. Therefore, due to the impact, the total mechanical energy of the system changes by $\Delta K$ so that on the time interval $(0, \infty)$,

$$
\frac{m \dot{x}^{2}}{2}+\frac{k|x|^{n+1}}{n+1}=\frac{k|a|^{n+1}}{n+1}+m\left[\frac{v_{0}^{2}}{2}+\dot{x}(-0) v_{0}\right], 0<t<\infty .
$$

From the relations of Eqs (42) and (43) it follows that

$$
\begin{aligned}
& \max _{t \in\left[-t_{0}, 0\right)}|x(t)|=|a|,|\dot{x}(t)| \leqslant \sqrt{\frac{2 k|a|^{n+1}}{m(n+1)}},-t_{0} \leqslant t<0, \\
& \max _{t \in[0, \infty)}|x(t)|=\left[|a|^{n+1}+\frac{m(n+1)}{k}\left(\frac{v_{0}^{2}}{2}+\dot{x}(-0) v_{0}\right)\right]^{\frac{1}{n+1}} .
\end{aligned}
$$

For fixed values of $a$ and $k$, choose the pre-action time $t_{0}$ (which uniquely determines the pre-impact velocity $\dot{x}(-0)$ so as to minimize the peak absolute value of the displacement of the object on the interval $0 \leqslant t<\infty$. From Eqs (44) and (45) it follows that this choice corresponds to

$$
\begin{aligned}
& \dot{x}(-0)=-\sqrt{\frac{2 k|a|^{n+1}}{m(n+1)}}, \\
& \max _{t \in[0, \infty)}|x(t)|=\left[|a|^{n+1}+\frac{m(n+1)}{k}\left(\frac{v_{0}^{2}}{2}-v_{0} \sqrt{\frac{2 k|a|^{n+1}}{m(n+1)}}\right)\right]^{\frac{1}{n+1}} .
\end{aligned}
$$

The relation of Eq. (47) implies that if

$$
k \geqslant \frac{(n+1) m v_{0}^{2}}{8|a|^{n+1}},
$$

then $\max _{t \in[0, \infty)}|x(t)| \leqslant|a|$ and, hence, in accordance with Eq. (44), we have $\max _{t \in\left[-t_{0}, \infty\right)}|x(t)|=|a|$. Otherwise, the inequality $\max _{t \in\left(-t_{0}, \infty\right)}|x(t)|>|a|$ holds. Therefore, the maximum of the quantity $\max _{t \in\left[-t_{0}, \infty\right)}|x(t)|$ for a fixed $a$ is attained for $k$ satisfying the inequality of Eq. (48) and is equal to $|a|$.

It follows that the optimal values of $k$ and $|a|$ are the solution of the system of inequalities

$$
k \geqslant \frac{(n+1) m v_{0}^{2}}{8|a|^{n+1}}, k|a|^{n} \leqslant U
$$

which corresponds to the minimum value of $|a|$. The first inequality of Eq. (49) repeats the relation of Eq. (48), while the second inequality expresses the constraint of Eq. (41), with reference to the fact that if the first inequality 
is satisfied, the maximum value of $|x(t)|$ is equal to $|a|$. The desired solution corresponds to the case where both of the relations of Eq. (49) hold as equalities and is defined by the expressions

$$
J\left(t_{0}, a_{0}, k_{0}\right)=\left|a_{0}\right|=\frac{(n+1) m v_{0}^{2}}{8 U}, k_{0}=\left[\frac{8}{(n+1) m v_{0}^{2}}\right]^{n} U^{n+1} .
$$

Substitute the expressions of Eq. (50) into the expressions of Eq. (46) for $a$ and $k$ to find the optimal value of the velocity of the object at the pre-impact instant

$$
\dot{x}(-0)=-v_{0} / 2 \text {. }
$$

From Eqs (50) and (51) it can be seen that for the optimal parameters, the kinetic energy of the system at the pre-impact instant is equal to the potential energy of the initially cocked spring, $\left(m \dot{x}^{2}(-0) / 2=k_{0}\left|a_{0}\right|^{n+1} /(n+1)=m v_{0}^{2} / 8\right)$ and, hence, is equal to the total energy of the system on the time interval before the impact. This implies that at the time of impact, the object is located at the position $x=0$ and the spring is unstrained.

To calculate the optimal value of the pre-action time use the relation of Eq. (42). Substitute $k=k_{0}$ and $|a|=\left|a_{0}\right|$ of Eq. (50) into Eq. (42) and introduce a new variable $\xi$ to represent this relation in the form

$$
\dot{\xi}^{2}=\left[\frac{4 U}{(n+1) m v_{0}}\right]^{2}\left(1-|\xi|^{n+1}\right), \xi=\frac{x}{\left|a_{0}\right|},-t_{0} \leqslant t<0 .
$$

From the initial conditions of Eq. (38) it follows that $\xi=\operatorname{sign} a_{0}$ for $t=-t_{0}$. As has been shown, for the optimal choice of the parameters, we have $x(0)=0$ and $\dot{x}(-0)=-v_{0} / 2$ and, accordingly, $\xi(0)=0$ and $\dot{\xi}(-0)=-v_{0} /\left(2\left|a_{0}\right|\right)$. Hence, the desired time instant $t_{0}=t_{0}^{*}$ is defined as an instant at which the object passes through the position $x=0$ with negative velocity for the first time. If $a_{0}>0$, then $\xi(0)=1$ and, hence, the derivative $\dot{\xi}$ is negative on the interval during which the object moves from the point $\xi=1$ to the point $\xi=0$. In this case, by integrating Eq. (52) with respect to $\xi$ from 1 to 0 and with respect to $t$ from $-t_{0}$ to 0 we obtain

$$
t_{0}=\frac{(n+1) m v_{0}}{4 U} \int_{0}^{1} \frac{d \xi}{\sqrt{1-\xi^{n+1}}}=\frac{m v_{0}}{4 U} \mathrm{~B}\left(\frac{1}{n+1}, \frac{1}{2}\right),
$$

where $\mathrm{B}(z, w)$ is the beta-function (Euler's integral of the first kind) [17].

If $a_{0}<0$, prior to arrival at the position $x=0$ with negative velocity for the first time, the object will move from $\xi=-1$ to $\xi=1$ with positive velocity and then from $\xi=1$ to $\xi=0$ with negative velocity. In this case

$$
t_{0}=\frac{3 m v_{0}}{4 U} \mathrm{~B}\left(\frac{1}{n+1}, \frac{1}{2}\right),
$$

which exceeds the value of Eq. (53) by a factor of 3. Hence, to minimize the pre-action time, the spring should be cocked in the positive direction.

Thus, the optimal values of the stiffness coefficient of the spring, the initial displacement of the object, and the pre-action time are given by

$$
k_{0}=\left[\frac{8}{(n+1) m v_{0}^{2}}\right]^{n} U^{n+1}, a_{0}=\frac{(n+1) m v_{0}^{2}}{8 U}, t_{0}^{*}=\frac{m v_{0}}{4 U} \mathrm{~B}\left(\frac{1}{n+1}, \frac{1}{2}\right) .
$$

The corresponding minimum of the peak absolute value of the displacement of the object to be protected is expressed as

$$
J\left(t_{0}^{*}, a_{0}, k_{0}\right)=\frac{(n+1) m v_{0}^{2}}{8 U} .
$$

A comparison of the relations of Eqs (13) and (56) indicates that the tuning of the spring with the power-law characteristic of Eq. (9) to the pre-acting operation enables one to decrease the value of the performance index by a factor of 4 , as compared with the operating mode without pre-action.

As was the case for the operating mode without pre-action, the quality of isolation in the case of pre-action is improved as the exponent of power of the spring characteristic, $n$, decreases. The best behavior corresponds to $n=0$. In this case, the pre-acting mode provides a decrease in the peak absolute value of the object's displacement by a factor of 4, as compared with the limiting performance for the control without pre-action. See Eq. (6). At the same time, the isolation quality provided by the optimal spring-based pre-acting isolator with power-law characteristic (for $n=0$ ) is by a factor of 2 inferior to the limiting performance of the isolator with pre-acting control. See Eq. (36). 


\section{Numerical examples}

Let the impact velocity, $v_{0}$, and the maximum acceleration allowed for the object to be protected, $U / m$, be defined as

$$
v_{0}=10 \mathrm{~m} / \mathrm{s}, U / m=100 \mathrm{~m} / \mathrm{s}^{2} .
$$

These data correspond approximately to the input data for the design of an isolation system for a helicopter seat to prevent the pilot from severe spinal injuries in the case of hard or crash landing. See [13,18].

The absolute minimum of the peak magnitude of the relative displacement of the object that can be achieved by the isolator without pre-action is defined by Eq. (6) and is given by

$$
J\left(u_{0}\right)=\frac{m v_{0}^{2}}{2 U}=50 \mathrm{~cm} .
$$

This quantity for the pre-acting isolator, in accordance with Eq. (36), is

$$
J\left(u_{0}, t_{0}^{*}\right)=\frac{m v_{0}^{2}}{16 U}=6.25 \mathrm{~cm} .
$$

From Eq. (13), the minimum value of the displacement peak magnitude that can be provided by a power-law spring without pre-action is

$$
J\left(k_{0}\right)=\frac{m v_{0}^{2}}{2 U}(n+1)=50(n+1) \mathrm{cm},
$$

and the corresponding value for the spring that has been cocked and then released, thereby implementing the pre-action concept, according to Eq. (56), is

$$
J\left(t_{0}^{*}, a_{0}, k_{0}\right)=\frac{m v_{0}^{2}}{8 U}(n+1)=12.5(n+1) \mathrm{cm},
$$

where $n$ is the exponent of the power-law characteristic of the spring Eq. (9).

The minimum pre-action time of the isolator providing the limiting performance characteristic is determined by Eq. (36) and for the input data of Eq. (57) is

$$
t_{0}^{*}=\frac{m v_{0}}{U}=0.1 \mathrm{~s}
$$

For an elastic power-law isolator, the pre-action time is defined by Eq. (55). The numerical values of this time for a bang-bang spring, corresponding to $n=0$, and a linear spring $(n=1)$ are

$$
n=0: t_{0}^{*}=\frac{m v_{0}}{2 U}=0.05 \mathrm{~s} ; \quad n=1: t_{0}^{*}=\frac{\pi m v_{0}}{4 U}=0.08 \mathrm{~s} .
$$

\section{Peak displacement and the rattlespace}

In this paper, we utilized the peak magnitude of the displacement of the object to be protected relative to the base as the performance index to be minimized. Depending on the specific problem to be solved, this criterion was defined by Eqs (4), (28), or (40). This criterion is commonly used to measure the stroke of the shock isolator in response to an impact and, thereby, the space needed between the object and the base (i.e., the rattlespace). See, for example [7-9,12-15]. However, it is of interest to show that the displacement peak magnitude is not always an adequate measure for the rattlespace.

Consider the limiting performance analysis of shock isolation without pre-action versus that with pre-action. As was stated in Section 2, the limiting performance of the isolation without pre-action can be provided by the control

$$
u_{0}(t)=\left\{\begin{array}{ll}
-U, & \text { if } 0 \leqslant t<m v_{0} / U \\
0, & \text { if } t \geqslant m v_{0} / U
\end{array} .\right.
$$

The respective time history of the relative displacement of the object is given by 


$$
x(t)=\left\{\begin{array}{ll}
v_{0} t-U t^{2} /(2 m), & \text { if } 0 \leqslant t<m v_{0} / U \\
m v_{0}^{2} /(2 U), & \text { if } t \geqslant m v_{0} / U
\end{array} .\right.
$$

When subject to the control of Eq. (64), the body starts moving at the instant of impact and continues to travel in the direction of the impact impulse until coming to a full stop at the position $x=m v_{0}^{2} /(2 U)$. Therefore, in this case, the rattlespace coincides with the peak displacement.

Consider now the relative motion of the object subject to the control of Eq. (30) that provides the limiting performance for the pre-acting isolation system. In accordance with Eqs (29) and (34) and Fig. 3, the object first travels the distance of $m v_{0}^{2} /(16 U)$ in the direction of the impact impulse, then the distance $m v_{0}^{2} /(8 U)$ in the backward direction, and then again the distance $m v_{0}^{2} /(8 U)$ in the direction of the impact impulse. As a result, the peak magnitude of the displacement is equal to $m v_{0}^{2} /(16 U)$, in accordance with Eqs. (36) and (59), whereas the actual rattlespace is measured by $m v_{0}^{2} /(8 U)$, which corresponds to $12.5 \mathrm{~cm}$ for the input data of Eq. (57). Therefore, although the maximum reduction in the displacement peak magnitude that can be achieved by the utilization of an isolator with pre-acting control, as compared with an isolator without pre-action, is characterized by a factor of 8 , the maximum reduction in the rattlespace is characterized by a factor of 4 .

The peak magnitude of the displacement of the body to be protected provided by spring isolators with optimal parameters is determined by Eq. (13) in the case where pre-action is absent and by Eq. (56) in the case of pre-action. As was the case above, the measure of the actual rattlespace is twice as large as that given by the respective values of $J\left(k_{0}\right)$ and $J\left(t_{0}^{*}, a_{0}, k_{0}\right)$. These values define the amplitude of oscillations of the object on the spring (the distance from the equilibrium position to one of the extreme positions of the oscillating body), whereas the total swing of these oscillations (the distance between two extreme positions of the body) exceeds the amplitude by a factor of 2 .

Since for elastic undamped isolators, as well as for the pre-acting isolator that provides the limiting performance, the rattlespace is two times the peak magnitude of the displacement, the ratios of the values of the peak displacements in theses cases coincides with those of the rattlespace.

In the general case, there is no simple relation between the maximum peak displacement and the rattlespace. For that reason, when it is the rattlespace that should be minimized by the design of the isolator, it is reasonable to replace the criterion

$$
J=\max _{t \in\left[-t_{0}, \infty\right)}|x(t)|
$$

by the difference between the maximum and minimum values of the relative displacement of the object during its motion, i.e., by the quantity

$$
\tilde{J}=\max _{t \in\left[-t_{0}, \infty\right)} x(t)-\min _{t \in\left(-t_{0}, \infty\right)} x(t) .
$$

The relations of Eqs (66) and (67) correspond to the pre-acting control, since the respective extrema are calculated on the interval $\left(-t_{0}, \infty\right)$. However, the control without pre-action can be regarded as a particular case of the pre-acting control for $t_{0}=0$. Hence, these relations apply to both pre-acting isolators and those without pre-action.

The performance index of Eq. (67) defines a new class of optimization problems for shock/impact isolators. To the authors' knowledge, such problems have not been identified previously.

We choose to present a pre-acting control which is different from the control of Eq. (30) that provides the limiting performance for the system of Eqs (31) and (32) with respect to the criterion of Eq. (66) but leads to the same rattlespace. However, the maximum peak displacement corresponding to this control will exceed that of Eq. (36) or Eq. (59). This control has the form

$$
u(t)=\left\{\begin{array}{ll}
-U, & \text { if }-t_{0} \leqslant t<t_{0} \\
0, & \text { if } t \geqslant t_{0}
\end{array}, t_{0}=\frac{m v_{0}}{2 U} .\right.
$$

This is a simple constant force control. It is switched on to act for $t_{0}$ time units before the impact and is switched off in $t_{0}$ time units after the impact. The corresponding time histories of the relative velocity, $\dot{x}(t)$, and the relative displacements, $x(t)$, of the object are defined by

$$
\dot{x}(t)=\left\{\begin{array}{ll}
-U\left(t+t_{0}\right) / m, & \text { if }-t_{0} \leqslant t<0 \\
v_{0}-U\left(t+t_{0}\right) / m, & \text { if } 0 \leqslant t<t_{0} \\
0, & \text { if } t \geqslant t_{0}
\end{array}, t_{0}=\frac{m v_{0}}{2 U}\right.
$$




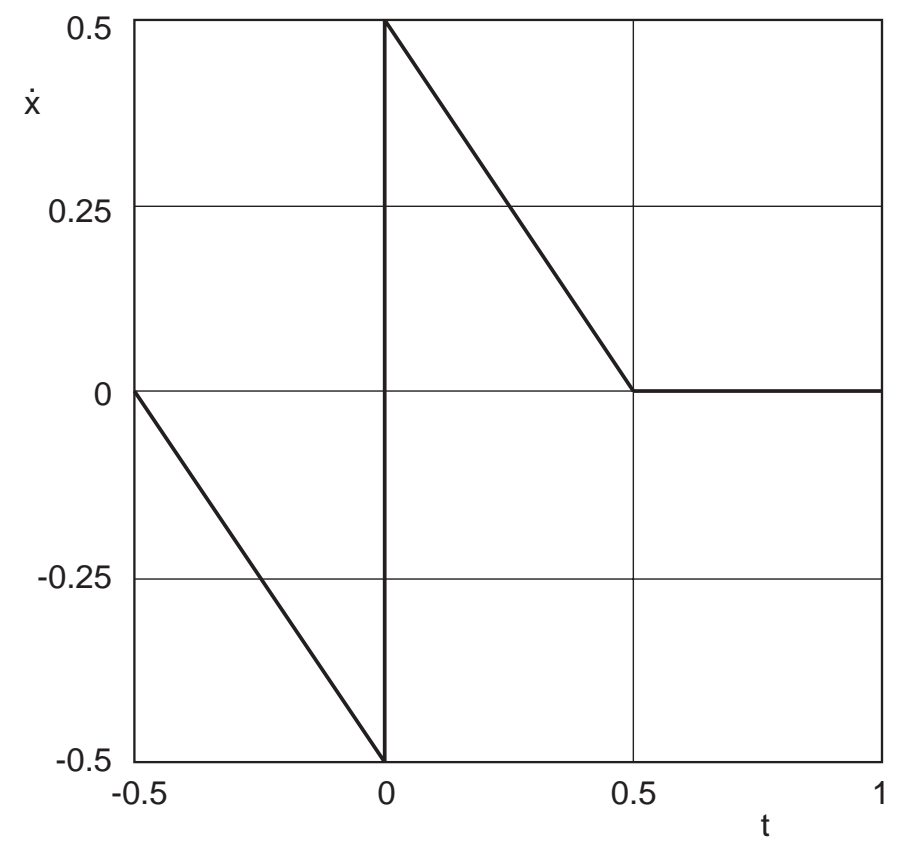

Fig. 5. Time history of the relative velocity of the object corresponding to the constant force pre-acting control.

$$
x(t)=\left\{\begin{array}{ll}
-U\left(t+t_{0}\right)^{2} /(2 m), & \text { if }-t_{0} \leqslant t<0 \\
v_{0} t-U\left(t+t_{0}\right)^{2} /(2 m), & \text { if } 0 \leqslant t<t_{0} \\
0, & \text { if } t \geqslant t_{0}
\end{array}, t_{0}=\frac{m v_{0}}{2 U} .\right.
$$

The time histories of Eqs (69) and (70) are plotted in Figs 5 and 6, respectively. The numerical values in these figures correspond to the dimensionless variables of Eq. (29), i.e., to the case of $m=1, v_{0}=1$, and $U=1$ in Eqs (69) and (70). It is apparent from Fig. 6 that

$$
\max _{t \in\left[-t_{0}, \infty\right)} x(t)=0, \min _{t \in\left[-t_{0}, \infty\right)} x(t)=-m v_{0}^{2} /(8 U), J=\tilde{J}=m v_{0}^{2} /(8 U)
$$

The peak magnitude of the relative displacement of the object provided by the control of Eq. (69) is two times that provided by the control of Eq. (30), but the rattlespaces corresponding to these two controls coincide.

\section{Conclusions}

In a number of cases, a pre-acting control can be utilized in shock/impact isolation systems. The pre-action concept implies that the controller is engaged in advance, i.e., before the base has been in fact subjected to an impact. The pre-acting control requires the time of initiation of the disturbance and its dynamic characteristics, in particular, the duration and the impact impulse, to be able to be predicted beforehand. The limiting performance analysis shows that the pre-acting control allows a substantial improvement in the quality of shock/impact isolation, as compared with the performance provided by the traditional isolators without pre-action. The isolators without pre-action are engaged simultaneously with the impact. In fact, such isolators are triggered by the impact as it occurs. For a single-degree-of-freedom system subjected to an instantaneous impact, a factor of 8 improvement is provided by the pre-acting control, when measured by the criterion of the peak magnitude of the displacement of the body to be protected relative to the base.

A substantial improvement in the performance of the isolation system as compared with the limiting performance of the isolation system without pre-action can be achieved by a commonly utilized elastic isolator (a spring), provided that it has been pre-strained and then is released at an appropriate instant before the impact. The optimal values of 


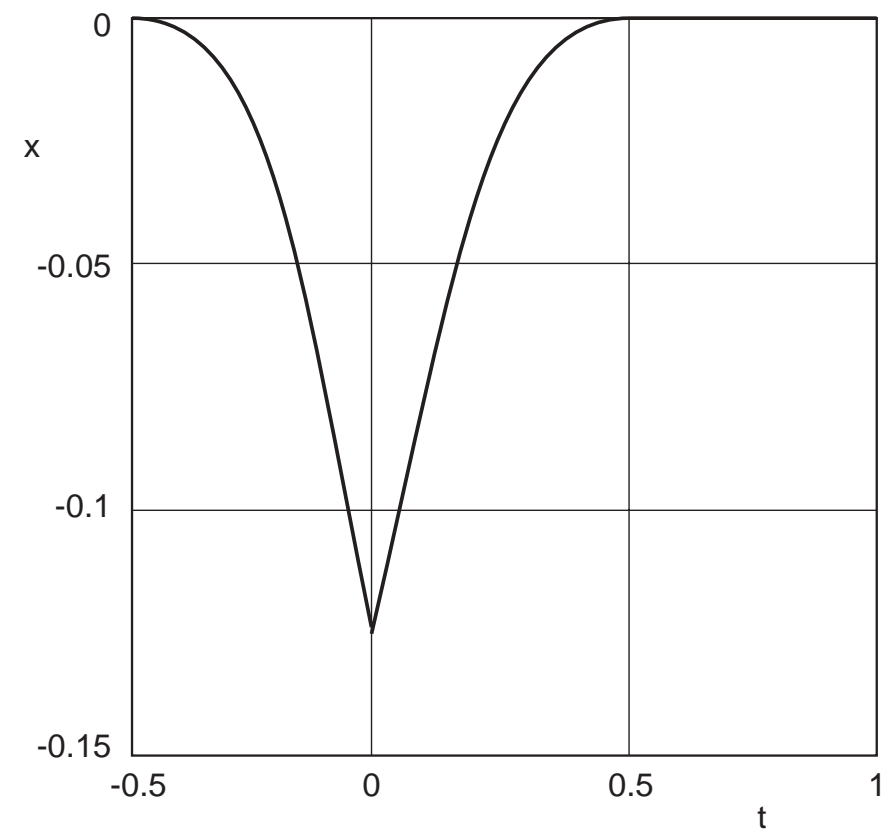

Fig. 6. Time history of the relative displacement of the object corresponding to the constant force pre-acting control.

the pre-strain magnitude, the release time, and the stiffness depend on the impact intensity and the mass of the object to be protected. For a single-degree-of-freedom system, the utilization of the pre-acting spring isolator leads to a decrease in the peak displacement of the object relative to the base by a factor of 4 , as compared with the limiting performance of the isolator without pre-action, without an increase in the peak magnitude of the force transmitted to the object by the isolator.

Although commonly used as a measure of the stroke of the shock isolator in response to an impact, the maximum absolute value of the displacement of the object to be protected relative to the base is not always a measure of the rattlespace, i.e., the space needed between the object and the base. If the object responding to an impact moves horizontally both to the left and right of its home position, the rattlespace exceeds the displacement magnitude peak. This is the case, for example, for pre-acting isolators considered in the present paper. In view of this, in the general case, it is reasonable to replace the criterion of the maximum absolute value of the relative displacement of the object by the difference between the maximum and minimum values of this displacement. This defines a different class of optimization problems for shock/impact isolators.

\section{Acknowledgments}

This research was partly supported by NSF (grant BES-0302337), NATO (grant PST.CLG.979409), and the Russian Foundation for Basic Research (grants 02-01-00157 and 04-01-00222).

\section{References}

[1] V.S. Anastasevich, The effectiveness of the protection of a device from shock by means of its isolation (in Russian), Inzhenernyi Sbornik, 1(1) (1941), 71-72.

[2] A. Yu. Ishlinskii, Mechanics of Gyro Systems (in Russian), Izd-vo AN SSSR, Moscow, 1968.

[3] A. Yu. Ishlinskii, Classical Mechanics and Inertial Forces (in Russian), Nauka, Moscow, 1987.

[4] E. Sevin, Min-max solutions for the linear mass-spring system, J. Applied Mechanics 24 (1957), 131-136.

[5] E. Sevin, On the design of shock isolated floor systems, Shock and Vibration Bulletin (1960), 22-35.

[6] M.Z. Kolovskii, Nonlinear Theory of Vibration Isolation Systems (in Russian), Nauka, Moscow, 1966. 
[7] M.Z. Kolovskii, Automatic Control of Vibration Isolation Systems (in Russian), Nauka, Moscow, 1976.

[8] V.V. Guretskii, On one problem of optimal control, Izv. AN SSSR. Mekhanika 1 (1965), 159-162.

[9] E. Sevin and W.D. Pilkey, Optimum Shock and Vibration Isolation, Shock and Vibration Information Analysis Center, Washington DC, 1971.

[10] K.V. Frolov, ed., Vibration in Engineering: A Handbook. Protection from Vibration and Shock (in Russian), Vol. 6, Mashinostroenie, Moscow, 1995.

[11] C.M. Harris and C.E. Crede, Shock and Vibration Handbook, McGrow-Hill, NY, 1996.

[12] D.V. Balandin, N.N. Bolotnik and W.D. Pilkey, Optimal protection from impact and shock: Theory and methods, Applied Mechanics Reviews 53(9) (2000), 237-264.

[13] D.V. Balandin, N.N. Bolotnik and W.D. Pilkey, Optimal Protection from Impact, Shock, and Vibration, Gordon and Breach Science Publishers, Amsterdam, 2001.

[14] N.N. Bolotnik, Optimization of Shock and Vibration Isolation Systems (in Russian), Nauka, Moscow, 1983.

[15] K.A. Afimiwala and R.W. Mayne, Optimal design of an impact absorber, Journal of Engineering for Industry 96(1) (1974), 124-130.

[16] V.V. Guretskii, On the problem of minimizing the maximum displacement (in Russian), Trudy LPI. Mekhanika $i$ Protsessy Upravleniya, Vychislitel'naya Matematika 307 (1969), 11-21.

[17] E. Janke, F. Emde and F. Loesch, Tafeln Hoeherer Funktionen, B.G. Teubner, ed., Verlagsgesellschaft, Stuttgart, 1960.

[18] Z.Q. Cheng, W.D. Pilkey, D.V. Balandin, N.N. Bolotnik, J.R. Crandall and C.G. Shaw, Optimal control of helicopter seat cushions for the reduction of spinal injuries, Intern. J. Crashworthiness 6(3) (2001), 321-338. 

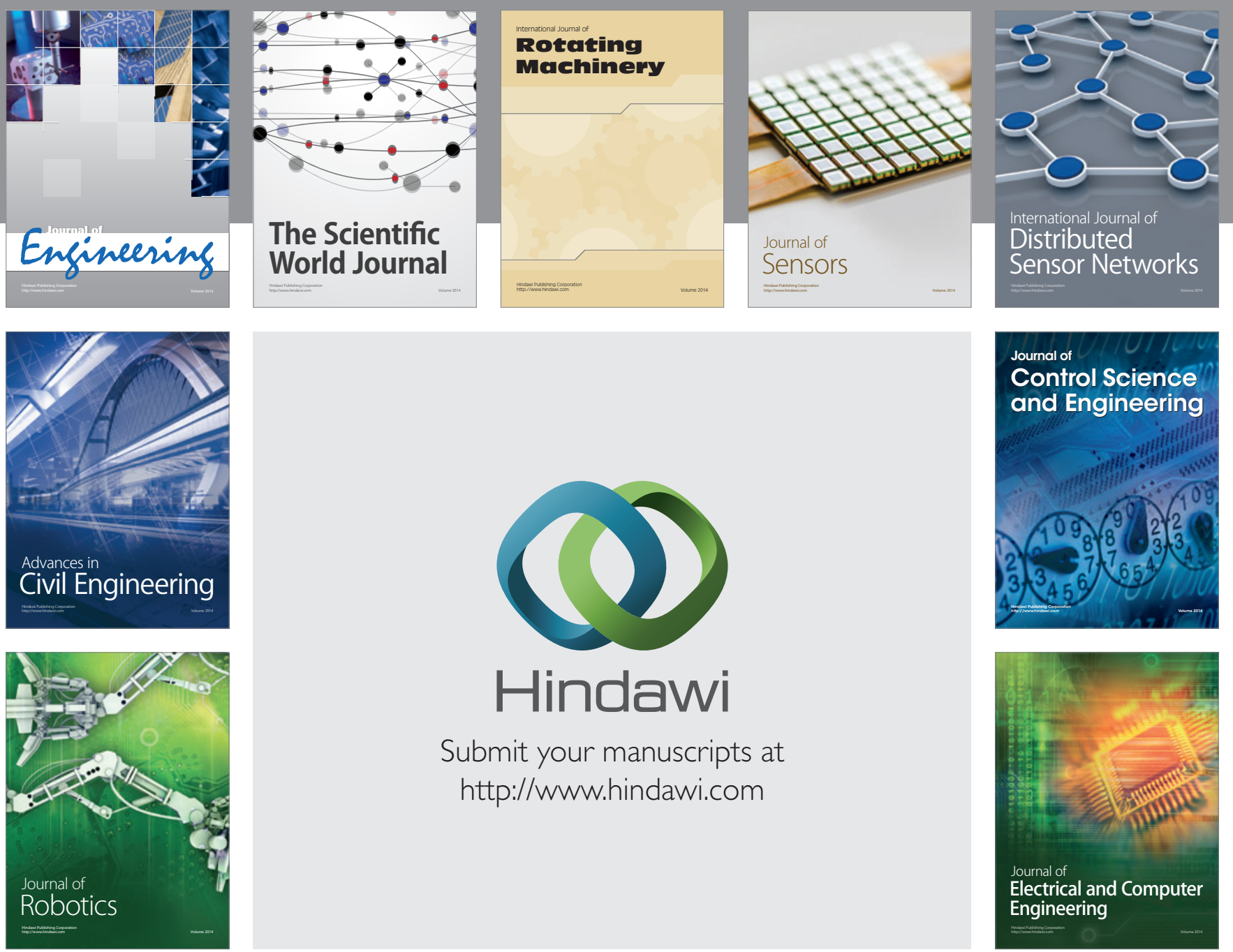

Submit your manuscripts at

http://www.hindawi.com
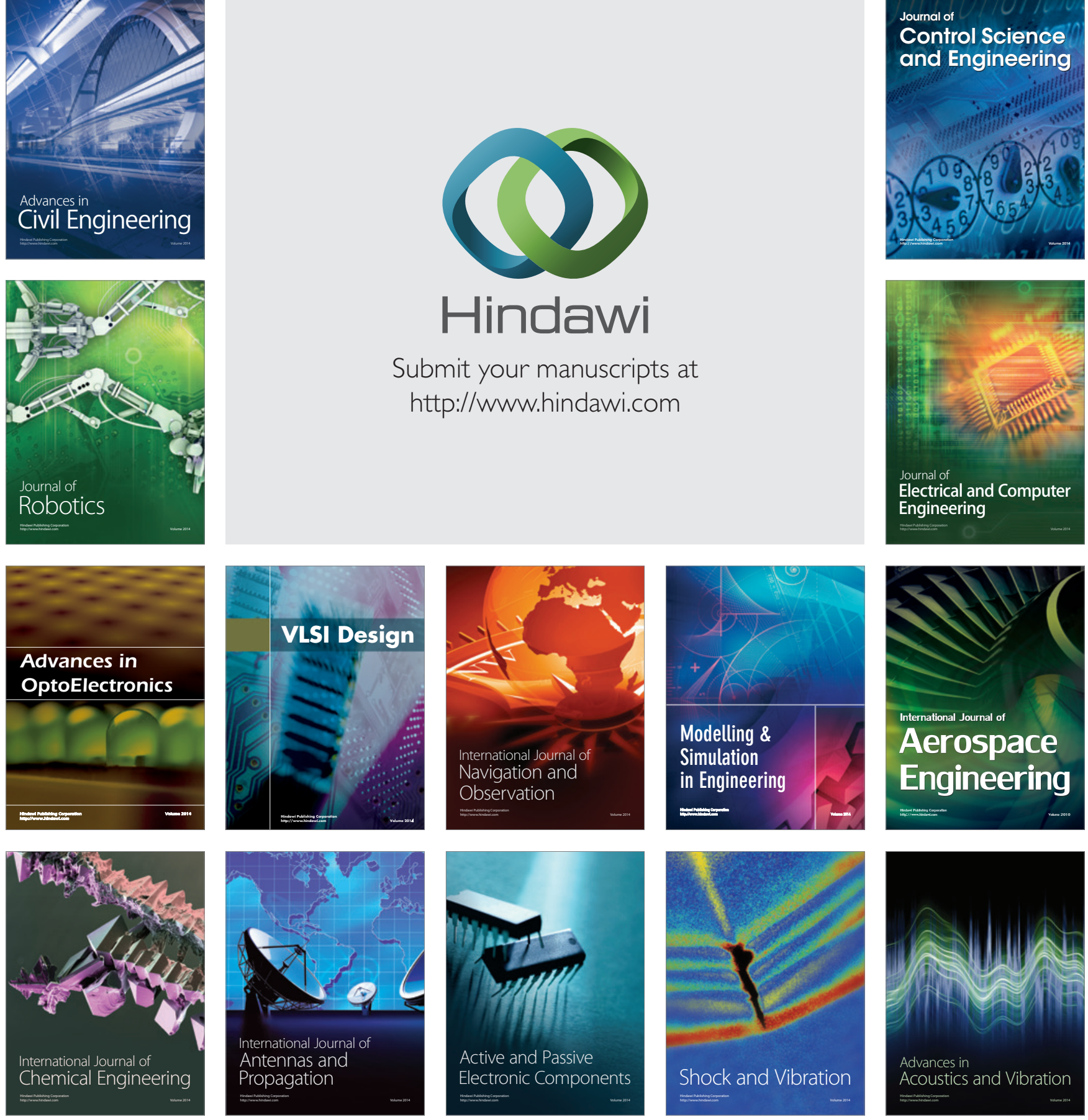